\title{
Correction to: The mystery of "missing" visits in an emergency cardiology department, in the era of COVID-19.; a time-series analysis in a tertiary Greek General Hospital
}

Konstantinos Tsioufis ${ }^{1}\left[\right.$ D . Christina Chrysohoou ${ }^{1} \cdot$ Maria Kariori $^{1} \cdot$ loannis Leontsinis ${ }^{1} \cdot$ Ioannis Dalakouras $^{1}$. Angelos Papanikolaou ${ }^{1} \cdot$ Georgios Charalambus ${ }^{2} \cdot$ Helen Sambatakou $^{3} \cdot$ Gerasimos Siasos $^{1}$.

Demosthenes Panagiotakos ${ }^{4}$. Dimitrios Tousoulis ${ }^{1}$

Published online: 7 August 2020

(c) Springer-Verlag GmbH Germany, part of Springer Nature 2020

Correction to: Clinical Research in Cardiology

https://doi.org/10.1007/s00392-020-01682-1

The original version of this article unfortunately contained a mistake. The spelling of Gerasimos Siasos's name was incorrect. The corrected author list is given above.

The original article has been corrected.

The original article can be found online at https://doi.org/10.1007/ s00392-020-01682-1.

Konstantinos Tsioufis

ktsioufis@hippocratio.gr

1 First Cardiology Clinic, Medical School, National and Kapodistrian University of Athens, Hippokration Hospital, 114 Vass Sofias Ave, 11527 Athens, Greece

2 Emergency Department, Hippocratio Hospital, Athens, Greece

3 2nd Department of Internal Medicine, HIV Unit, Medical School, Hippokration General Hospital, National and Kapodistrian University of Athens, Athens, Greece

4 School of Health Science and Education, Harokopio University, Athens, Greece 